TRANSACTIONS OF THE

AMERICAN MATHEMATICAL SOCIETY

Volume 349, Number 12, December 1997, Pages 5001-5019

S $0002-9947(97) 01831-\mathrm{X}$

\title{
PARTITION IDENTITIES INVOLVING GAPS AND WEIGHTS
}

\author{
KRISHNASWAMI ALLADI
}

\begin{abstract}
We obtain interesting new identities connecting the famous partition functions of Euler, Gauss, Lebesgue, Rogers-Ramanujan and others by attaching weights to the gaps between parts. The weights are in general multiplicative. Some identities involve powers of 2 as weights and yield combinatorial information about some remarkable partition congruences modulo powers of 2 .
\end{abstract}

\section{INTRODUCTION}

Four of the most fundamental and extensively studied partition functions are:

(i) $p(n)=$ the number of unrestricted partitions of $n$,

(ii) $Q(n)=$ the number of partitions of $n$ into distinct parts,

(iii) $g(n)=$ the number of partitions of $n$ in which the even parts do not repeat, and

(iv) $\rho(n)=$ the number of partitions of $n$ with difference $\geq 2$ between parts.

In laying the foundations of the theory of partitions, Euler established several generating function identities involving $p(n)$ and $Q(n)$. The function $g(n)$ appears in a famous identity due to Lebesgue and Gauss. Finally, $\rho(n)$ is the function appearing in the celebrated (first) Rogers-Ramanujan identity. Andrews' encyclopedia [5] contains a wealth of information about these and related partition functions.

Our purpose here is to provide some new relationships for these classical partition functions by attaching weights which are defined multiplicatively using gaps between parts. Such weighted identities have many applications, a few of which are briefly discussed here. Certain applications of great interest are discussed in detail elsewhere (see [1], [2]). For instance, the most striking application (see [1]) is a combinatorial correspondence for a deep partition theorem of Göllnitz [11], which is obtained by suitably extending the weighted identity in Theorem 15 of $\S 7$ and interpreting this extension combinatorially.

To understand these weighted identities in a proper setting, consider the following general problem: Given a set $S$ of partitions, let $P_{S}(n)$ denote the number of partitions $\pi$ of $n$ with $\pi \in S$. Suppose $S \subseteq T$. Our problem is to determine weights

Received by the editors October 10, 1995 and, in revised form, June 3, 1996.

1991 Mathematics Subject Classification. Primary 11P83, 11P81; Secondary 05A19.

Key words and phrases. Partitions, weights, gaps, Durfee squares, powers of 2.

Research supported in part by the National Science Foundation Grant DMS 9400191.

(C)1997 American Mathematical Society 
$w_{S}(\pi) \geq 1$ in a natural way such that for all $n$

$$
P_{T}(n)=\sum_{\substack{\sigma(\pi)=n \\ \pi \in S}} w_{S}(\pi) .
$$

(Here and in what follows, $\sigma(\pi)$ is the sum of the parts of $\pi$; for further notation see $\S 2$.) Note that determining weights $w_{T}(\pi)$ such that

$$
P_{S}(n)=\sum_{\substack{\sigma(\pi)=n \\ \pi \in T}} w_{T}(\pi)
$$

is trivial, because $S \subseteq T$ implies that

$$
w_{T}(\pi)= \begin{cases}1, & \text { if } \pi \in S, \\ 0, & \text { if } \pi \notin S,\end{cases}
$$

is a valid choice. One may view (1.1) as some sort of an "inversion" of (1.2), but no such general inversion formula is known. For most choices of $S$ and $T$, finding the weights $w_{S}(\pi)$ is quite difficult. Our primary purpose here is to produce such weights when $P_{S}(n)$ and $P_{T}(n)$ are the classical functions listed above.

After introducing notation and partition preliminaries in $\S 2$, a weighted identity connecting $P_{S}(n)=\rho(n)$ and $P_{T}(n)=p(n)$ is derived in $\S 3$ (see Theorem 1). The Rogers-Ramanujan partition function has another interpretation; namely, $\rho(n)$ is the number of partitions of $n$ where each part is $\geq$ the number of parts. This second interpretation yields the identity in Theorem $2, \S 3$, which has the advantage that it extends to the case where $P_{T}(n)=p(n)$ and $P_{S}(n)$ is the number of partitions of $n$ into fewer than $k$ Durfee squares (see Theorem 8, §4).

Next, by utilizing a variation in a combinatorial technique due to Bressoud [9], a weighted identity connecting $Q(n)$ and $\rho(n)$ in derived in $\S 5$.

In terms of applications, the results of $\S \S 6$ and 7 are the most significant. Section 6 discusses a result proved by Alladi and Gordon [4] connecting $Q(n)$ and $g(n)$ via the identity

$$
g(n)=\sum_{k} Q(n, k) 2^{k}
$$

and its refinement. Here $Q(n, k)$ is the number of partitions $b_{1}+b_{2}+\cdots+b_{\nu}$ counted by $Q(n)$ with the added restriction that there are exactly $k$ gaps $b_{i}-b_{i+1}$ which are $\geq 2$ with the convention that $b_{\nu+1}=0$. One reason (1.4) is of interest is because it indicates that $g(n)$ is almost always a multiple of any given power of 2 . In $\S 7$, a similar result is derived for $Q(n)$, a result I had proved in 1990, namely,

$$
Q(n)=\sum_{k} g_{3}(n, k) 2^{k} .
$$

For a precise definition of $g_{3}(n, k)$, see $\S 7$. I had proved (1.5) by a combinatorial study of an identity of Sylvester [16] in his classic paper of 1882, and this proof is given in $\S 7$. Identity (1.5) suggests the remarkable result that for each positive integer $k$, the congruence

$$
Q(n) \equiv 0\left(\bmod 2^{k}\right)
$$

holds for almost all values of $n$. A proof of (1.6) and an extension of it to $p$ regular partitions has been given by Gordon and Ono [13] making use of the theory of modular forms. Naturally it is of interest to give a combinatorial proof of the 
validity of (1.6) for almost all $n$, and (1.5) appears to be the key to the combinatorial understanding of (1.6). Similarly, (1.4) is the key to the combinatorial study of the related congruence

$$
g(n) \equiv 0\left(\bmod 2^{k}\right)
$$

In a forthcoming paper [2] we utilize (1.4) and (1.5) to show combinatorially that for small $k,(1.6)$ and (1.7) are valid almost always, and to determine the exceptional sets of zero density where the congruences fail.

Recently, Bowman [8] has obtained many interesting results for various classical partition functions by inserting 'ones' in gaps. Bowman's notions are different from ours and so are his results, which are obtained by a study of continued fractions. But there appear to be connections between his approach and ours which may be worth a deeper study.

\section{PARTition PRELIMINARIES}

Given any complex number $a$ and a positive integer $n$, set

$$
(a)_{n}=(a ; q)_{n}=\prod_{n=0}^{n-1}\left(1-a q^{j}\right)
$$

and for $|q|<1$,

$$
(a)_{\infty}=\lim _{n \rightarrow \infty}(a)_{n}=\prod_{j=0}^{\infty}\left(1-a q^{j}\right) .
$$

We use the symbol $\pi$ to denote a partition as well as its Ferrers graph. The conjugate of the Ferrers graph of $\pi$ is denoted by $\pi^{*}$.

The Ferrers graph of any partition $\pi$ contains a largest square $D_{1}(\pi)=D_{1}$ of nodes starting from the left hand top corner as is shown in Figure 1. This is the Durfee square of the Ferrers graph. The nodes to the right of $D_{1}(\pi)$ constitute a partition denoted by $\pi_{r}$. Similarly, the nodes below $D_{1}(\pi)$ constitute another partition denoted by $\pi_{b}$.

Given a partition $\pi: b_{1}+b_{2}+\cdots+b_{\nu}$ with $b_{1} \geq b_{2} \geq \cdots \geq b_{\nu}>0$, we let $\sigma(\pi)$ denote the sum of the parts of $\pi$ and $\nu(\pi)=\nu$, the number of parts of $\pi$. Also $\nu^{*}(\pi)$ is the number of different parts of $\pi$ (not counting repetitions) and $\lambda(\pi)=b_{1}$,

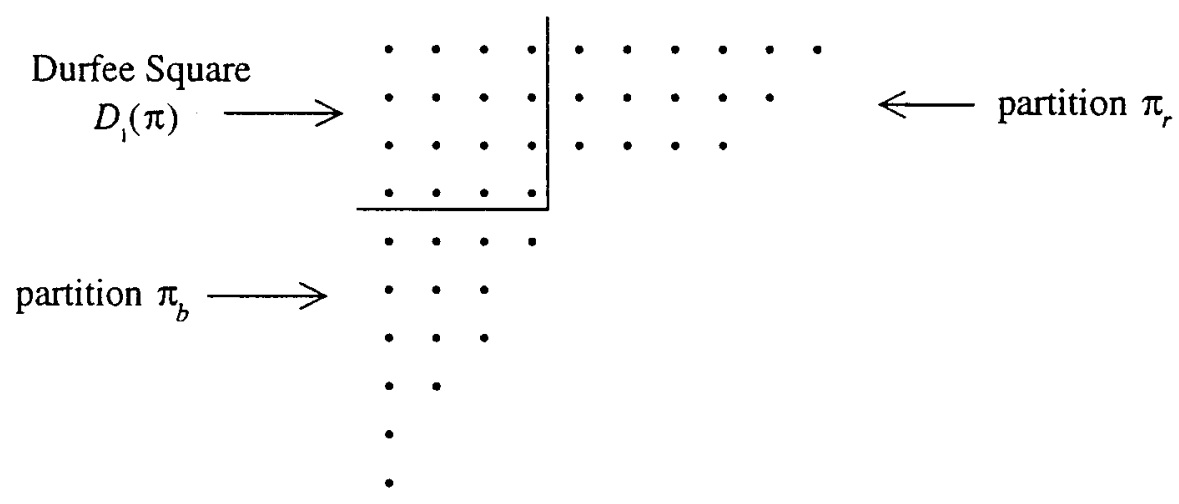

Figure 1 
the largest part of $\pi$. Sometimes we need to keep track of parts of a certain type, and this will be indicated by a subscript.

Given two partitions $\pi_{1}$ and $\pi_{2}$, by $\pi_{1}+\pi_{2}$ we mean the partition whose parts are obtained by adding the number of nodes in the corresponding rows of the Ferrers graphs of $\pi_{1}$ and $\pi_{2}$.

Since each partition $\pi$ may be viewed as a multi-set whose elements are positive integers arranged in decreasing order, we define $\pi_{1} \cup \pi_{2}$ to be the partition obtained by considering the set theoretic union of $\pi_{1}$ and $\pi_{2}$.

For each integer $j \geq 1$, the function

$$
1+\frac{b z q^{j}}{1-z q^{j}}=\frac{1+z(1-b) q^{j}}{1-z q^{j}}
$$

is the generating function for partitions $\pi$ using only the part $j$, where each nonempty partition is counted with weight $b z^{\nu(\pi)}$. Thus

$$
\frac{(-z(1-b) q)_{n}}{(z q)_{n}}=\sum_{\lambda(\pi) \leq n} b^{\nu^{*}(\pi)} z^{\nu(\pi)} q^{\sigma(\pi)}
$$

is the generating function for partitions $\pi$ with largest part $\leq n$, with the power of $b$ keeping track of the number of different parts and the power of $z$ counting the total number of parts.

For a partition $\pi$ whose parts are $b_{1} \geq b_{2} \geq \cdots \geq b_{\nu}>0$, the gaps in the partition are the differences $b_{i}-b_{i+1}$ for $i=1,2, \ldots, \nu$, where $b_{\nu+1}$ will be defined suitably based on the type of partition being discussed. For instance, in $\S 6$ we take $b_{\nu+1}=0$, whereas in $\S 7, b_{\nu+1}=-1$.

\section{UnRESTRICTED AND ROgers-RAMANUJAN PARTITIONS}

One of the fundamental identities that Euler proved for the number of unrestricted partitions of $n$ is

$$
\sum_{n=0}^{\infty} p(n) q^{n}=\sum_{\nu=0}^{\infty} \frac{q^{\nu^{2}}}{(q)_{\nu}^{2}}=\frac{1}{(q)_{\infty}} .
$$

Identity (3.1) is best understood by appeal to Durfee squares as in [14].

The celebrated first Rogers-Ramanujan identity is

$$
\sum_{\nu=0}^{\infty} \frac{q^{\nu^{2}}}{(q)_{\nu}}=\frac{1}{\left(q ; q^{5}\right)_{\infty}\left(q^{4} ; q^{5}\right)_{\infty}} .
$$

The combinatorial interpretation of the product on the right of (3.2) is clear: it is the generating function of partitions into parts $\equiv \pm 1(\bmod 5)$. One combinatorial interpretation for the left hand side is that it is the generating function of $\rho(n)$, the number of partitions of $n$ into parts differing by $\geq 2$. More specifically,

$$
\frac{q^{\nu^{2}}}{(q)_{\nu}}
$$

is the generating function for partitions into $\nu$ parts differing by $\geq 2$. Another interpretation for the term in (3.3) is that it is the generating function for partitions with a $\nu \times \nu$ Durfee square such that there are no nodes below the Durfee square as shown in Figure 2. That is, the partition $\pi_{b}$ (see Figure 1) does not exist here. We call such partitions as primary partitions. 


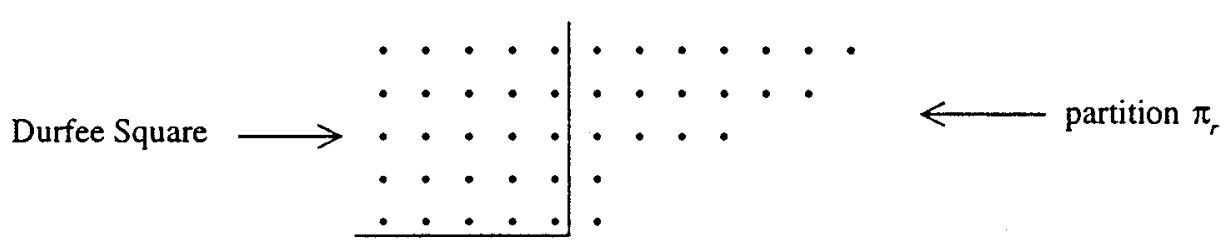

Figure 2

For primary partitions, the number of nodes in each row is at least as large as the number of rows. Thus $\rho(n)$ is also the number of partitions of $n$ into parts each at least as large as the number of parts. To get a bijection between partitions $\pi_{1}$ of $n$ into parts $b_{1} \geq b_{2} \geq \cdots \geq b_{\nu}$ with $b_{\nu} \geq \nu$ and partitions $\tilde{\pi}$ of $n$ into $\nu$ parts $h_{1}+h_{2}+\cdots+h_{\nu}$ differing by $\geq 2$, just count the number of nodes along hooks in the Ferrers graphs of the primary partitions. For the graph in Figure 2, the partition with minimal difference 2 that it generates is $16+13+9+4+2$ by counting nodes along hooks. In view of this bijection, and for later use, it is convenient to introduce the notation $\rho(\pi)=\tilde{\pi}$ to mean the partition obtained by counting nodes along hooks of the Ferrers graph of $\pi$.

Consider the decomposition

$$
\frac{q^{\nu^{2}}}{(q)_{\nu}^{2}}=\frac{q^{\nu^{2}}}{(q)_{\nu}} \cdot \frac{1}{(q)_{\nu}}
$$

To obtain a combinatorial interpretation of this decomposition, we define the sliding operation on Ferrers graphs.

Given a Ferrers graph $\pi$ corresponding to a partition of $n$, by a sliding operation $\psi$ on $\pi$ we mean the removal of certain columns to the right of the Durfee square of $\pi$ and the placement of these columns as rows below the Durfee square so as to form another Ferrers graph $\pi^{\prime}$. Several sliding operations $\psi$ can be performed on each partition $\pi$. We write $\pi^{\prime}=\psi(\pi)$ if $\pi^{\prime}$ is obtained from $\pi$ by a certain sliding operation. The following are invariants under the operation $\psi$.

(i) The total number of nodes in $\pi$ and $\pi^{\prime}$ are the same, that is, $\sigma(\pi)=\sigma\left(\pi^{\prime}\right)$.

(ii) The partitions $\pi$ and $\pi^{\prime}$ have the same Durfee square. That is, $D_{1}(\pi)=$ $D_{1}\left(\pi^{\prime}\right)$.

(iii) The sum of the number of parts and the largest part is invariant. That is,

$$
\nu(\pi)+\lambda(\pi)=\nu\left(\pi^{\prime}\right)+\lambda\left(\pi^{\prime}\right) .
$$

(iv) Most importantly, the partition obtained by counting nodes along the hooks of $\pi$ and $\pi^{\prime}$ will be the same. That is,

$$
\rho(\pi)=\rho\left(\pi^{\prime}\right) .
$$

In the partition displayed in Figure 3 , up to $d_{3}=3$ columns of length 3 could be moved from the right of the Durfee square and placed below the square. More generally, given a primary partition $\pi$ as in Figure 2, with parts $b_{1}, b_{2}, \ldots, b_{\nu}$, let $h_{1}, h_{2}, \ldots, h_{\nu}$ be the parts of $\tilde{\pi}=\rho(\pi)$ satisfying $h_{i}-h_{i+1} \geq 2$, for $1 \leq i \leq \nu-1$. Now write $h_{i}-h_{i+1}=2+d_{i}$. Notice that up to $d_{i}$ vertical columns of length $i$ could be removed from the right of the $D(\pi)$ and placed below the Durfee square as rows. Thus the number of choices of moving columns of length $i$ is $d_{i}+1=h_{i}-h_{i+1}-1$, for $1 \leq i \leq \nu-1$. For $i=\nu$, up to $h_{\nu}-1$ columns of length $\nu$ could be moved. 


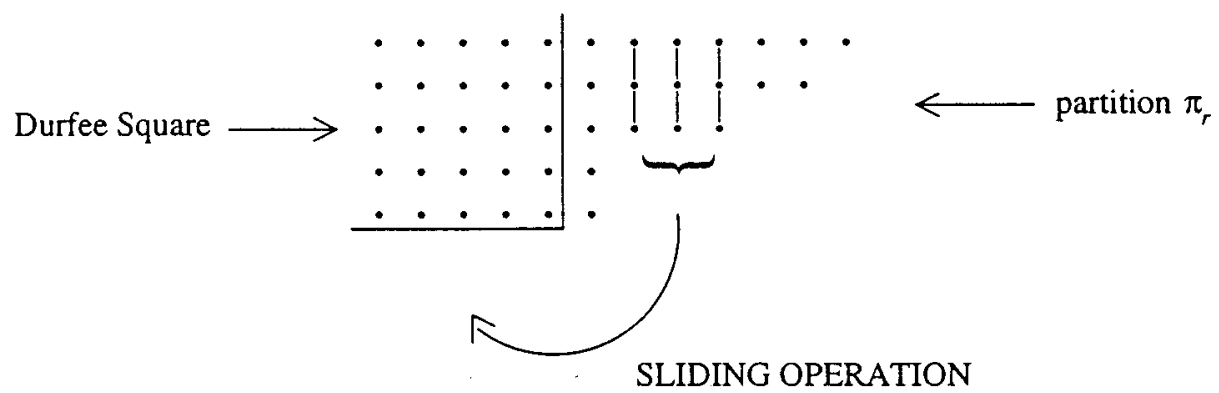

The 3 indicated columns could

be moved below the Durfee square

FiguRE 3

Thus the number of choices here is $h_{\nu}$. So this leads us to define a weight function for Rogers-Ramanujan partitions $\tilde{\pi}$ as follows:

$$
w_{R}(\tilde{\pi})=\prod_{i=1}^{\nu-1}\left(h_{i}-h_{i+1}-1\right) \cdot h_{\nu} .
$$

In view of the invariant (iv) above, we see that under the sliding operation each Rogers-Ramanujan partition $\tilde{\pi}$ of $n$ generates $w_{R}(\tilde{\pi})$ unrestricted partitions of $n$, and that all partitions of $n$ can be generated in this manner. So we have proved

Theorem 1. Let $R$ denote the set of all partitions with minimal difference 2. Then

$$
p(n)=\sum_{\substack{\sigma(\tilde{\pi})=n \\ \tilde{\pi} \in R}} w_{R}(\tilde{\pi}) .
$$

If we define $h_{\nu+1}=-1$, then the weights in (3.5) could be cast in the more elegant form

$$
w_{R}(\tilde{\pi})=\prod_{i=1}^{\nu}\left(h_{i}-h_{i+1}-1\right) .
$$

The connection between the parts $b_{i}$ of a primary partition $\pi$ and the parts $h_{i}$ of the partition $\tilde{\pi}=\rho(\pi)$ is

$$
b_{i}=h_{i}-(2(\nu-i)+1), \quad \text { for } \quad 1 \leq i \leq \nu .
$$

Now (3.7) implies that

$$
b_{i}-b_{i+1}+1=h_{i}-h_{i+1}-1, \quad \text { for } \quad 1 \leq i \leq \nu,
$$

with the conventions $b_{\nu+1}=\nu$ and $h_{\nu+1}=-1$. Thus under the sliding operation each primary partition $\pi$ with parts $b_{1}, b_{2}, \ldots, b_{\nu}$ generates $w_{1}(\pi)$ unrestricted partitions, where

$$
w_{1}(\pi)=\prod_{i=1}^{\nu}\left(b_{i}-b_{i+1}+1\right)
$$

(Compare (3.9) with (3.6).) This leads to Theorem 2 below, which may be viewed as a companion to Theorem 1. 
Theorem 2. Let $D_{1}$ denote the set of all partitions each of whose parts is at least as large as the number of parts. Then

$$
p(n)=\sum_{\substack{\sigma(\pi)=n \\ \pi \in D_{1}}} w_{1}(\pi) .
$$

The invariants (ii) and (iii) under the sliding operation lead to refinements of Theorem 1 and 2 as stated below.

Theorem 3. Let $p(n ; \nu)$ denote the number of partitions of $n$ having a $\nu \times \nu$ Durfee square.

Let $R_{\nu}$ and $D_{1, \nu}$ denote the subsets of $R$ and $D_{1}$ consisting of partitions having exactly $\nu$ parts. Then

$$
p(n ; \nu)=\sum_{\substack{\sigma \tilde{\pi})=n \\ \tilde{\pi} \in R_{\nu}}} w_{R}(\tilde{\pi})=\sum_{\substack{\sigma(\pi)=n \\ \pi \in D_{1, \nu}}} w_{1}(\pi) .
$$

Theorem 4. Let $p_{m}(n)$ denote the number of partitions $\pi$ of $n$ such that $\lambda(\pi)+$ $\nu(\pi)=m+1$.

Let $R(m)$ denote the subset of $R$ consisting of partitions $\tilde{\pi}$ for which $\lambda(\tilde{\pi})=m$.

Let $D_{1}(m)$ denote the subset of $D_{1}$ consisting of partitions $\pi$ such that $\lambda(\pi)+$ $\nu(\pi)=m+1$. Then

$$
p_{m}(n)=\sum_{\substack{\sigma(\tilde{\pi})=n \\ \tilde{\pi} \in R(m)}} w_{R}(\tilde{\pi})=\sum_{\substack{\sigma(\pi)=n \\ \pi \in D_{1}(m)}} w_{1}(\pi) .
$$

\section{Partitions into $<k$ Durfee squares}

The partition theoretic form of the first Rogers-Ramanujan identity (3.2) is

Theorem 5. The number of partitions $\rho(n)$ of $n$ into parts with minimal difference 2 is equal to the number of partitions of $n$ into parts $\equiv \pm 1(\bmod 5)$.

As was noticed earlier, $\rho(n)$ is also the number of partitions of $n$ into parts each at least as large as the number of parts.

The second Rogers-Ramanujan identity is

$$
\sum_{n=0}^{\infty} \frac{q^{n^{2}+n}}{(q)_{n}}=\frac{1}{\left(q^{2} ; q^{5}\right)_{\infty}\left(q^{3} ; q^{5}\right)_{\infty}} .
$$

This has the following combinatorial interpretation.

Theorem $5^{\prime}$. The number of partitions $\rho^{\prime}(n)$ into parts $\geq 2$ and with minimal difference 2 equals the number of partitions of $n$ into parts $\equiv \pm 2(\bmod 5)$.

In this case $\rho^{\prime}(n)$ is also equal to the number of partitions of $n$ into parts each of which is greater than the number of parts.

In the 1960's, Gordon [12] obtained the following significant extension of Theorems 5 and $5^{\prime}$ to an odd modulus $2 k+1$.

Theorem 6. Let $i$ and $k$ be integers satisfying $1 \leq i \leq k$ and $k>1$. Then the number $\rho_{k, i}(n)$ of partitions of $n$ in the form $\pi=h_{1}+h_{2}+\cdots+h_{\nu}$ such that $h_{j}-h_{j+k-1} \geq 2$ and with $\pi$ having fewer than $i$ ones equals the number of partitions of $n$ into parts $\not \equiv 0, \pm i(\bmod 2 k+1)$. 
Theorem 5 is the case $k=2, i=2$ and Theorem $5^{\prime}$ the case $k=2, i=1$ in Theorem 6.

Extending the interpretation of $\rho(n)$ in terms of Durfee squares, Andrews [6] showed

Theorem 7. The function $\rho_{k, k}(n)$ is equal to the number of partitions of $n$ whose Ferrers graphs have fewer than $k$ successive Durfee squares.

Successive Durfee squares in a partition $\pi=\pi_{1}$ can be defined as follows. We already know what the Durfee square of $\pi$ is. We will refer to this as the first Durfee square of $\pi=\pi_{1}$ and denote this by $D_{1}(\pi)=D_{1}\left(\pi_{1}\right)$. The portion of the Ferrers graph below $D_{1}(\pi)$ can be considered to be another Ferrers graph $\pi_{2}$ which has its own Durfee square $D_{1}\left(\pi_{2}\right)$. We call this the second Durfee square of $\pi_{1}$ and denote it by $D_{2}\left(\pi_{1}\right)$. The portion of the Ferrers graph below $D_{2}\left(\pi_{1}\right)$ is another Ferrers graph $\pi_{3}$ with its own Durfee square $D_{1}\left(\pi_{3}\right)=D_{3}\left(\pi_{1}\right)$, the third Durfee square of $\pi_{1}$, and so on. Thus each Ferrers graph $\pi_{1}$ has its own sequence of successive Durfee squares $D_{k}\left(\pi_{1}\right)$. In this sense, $\rho(n)=\rho_{2,2}(n)$ is equal to the number of partitions of $n$ whose Ferrers graphs have fewer than two Durfee squares. Partitions having at least two Durfee squares may be called non-Rogers-Ramanujan partitions as in $[10]$.

A natural question to ask at this point is whether there is a weighted identity connecting $p(n)$ with the partitions counted by $\rho_{k, k}(n)$. The answer to this is in the affirmative, as shown below.

A partition into $k$ successive Durfee squares can be written in the form

$$
\begin{aligned}
\pi= & b_{1}+b_{2}+\cdots+b_{\nu_{1}} \\
& +b_{\nu_{1}+1}+b_{\nu_{1}+2}+\cdots+b_{\nu_{1}+\nu_{2}} \\
& +b_{\nu_{1}+\nu_{2}+1}+b_{\nu_{1}+\nu_{2}+2}+\cdots+b_{\nu_{1}+\nu_{2}+\nu_{3}} \\
& \cdot \\
& \cdot \\
& +b_{\nu_{1}+\nu_{2}+\cdots+\nu_{k-1}+1}+b_{\nu_{1}+\nu_{2}+\cdots+\nu_{k-1}+2}+b_{\nu_{1}+\nu_{2}+\cdots+\nu_{k}}
\end{aligned}
$$

with the conditions

$$
b_{\nu_{1}} \geq \nu_{1}, b_{\nu_{1}+\nu_{2}} \geq \nu_{2}, \ldots, b_{\nu_{1}+\nu_{2}+\cdots+\nu_{k}} \geq \nu_{k} .
$$

Here $\nu_{1} \geq \nu_{2} \geq \cdots \geq \nu_{k}$ denote the sizes of the successive Durfee squares of $\pi$.

Consider the parts $b_{1}, b_{2}, \ldots, b_{\nu_{k}}$. Observe that if $1 \leq i<\nu_{k}$, then up to $b_{i}-b_{i+1}$ columns of length $i$ lying to the right of $D_{1}(\pi)$ could be extracted and placed horizontally below $D_{k}(\pi)$ to form a partition with $\geq k$ Durfee squares. For $i=\nu_{k}$, up to $b_{\nu_{k}}-b_{\nu_{k}+1}$ columns of length $\nu_{k}$ could be extracted if $\nu_{k}<\nu_{1}$ or up to $b_{\nu_{k}}-\nu_{k}$ columns of length $\nu_{k}$ could be extracted if $\nu_{k}=\nu_{1}$. These extracted columns are to be placed horizontally below $D_{k}(\pi)$ to form a Ferrers graph with $\geq k$ Durfee squares. Now define the weights $w_{k}(\pi)$ as follows:

$$
w_{k}(\pi)= \begin{cases}0, & \text { if } \pi \text { has }>k \text { Durfee squares } \\ 1, & \text { if } \pi \text { has }<k \text { Durfee squares }\end{cases}
$$

and for partitions $\pi$ with exactly $k$ Durfee squares

$$
w_{k}(\pi)=\prod_{i=1}^{\nu_{k}}\left(b_{i}-b_{i+1}+1\right), \text { if } \nu_{k}<\nu_{1},
$$


or

$$
w_{k}(\pi)=\left(b_{1}-b_{2}+1\right)\left(b_{2}-b_{3}+1\right) \ldots\left(b_{\nu_{k}-1}-b_{\nu_{k}}+1\right)\left(b_{\nu_{k}}-\nu_{k}+1\right) \text {, if } \nu_{k}=\nu_{1} .
$$

So what we have observed above is that every partition $\pi$ of $n$ in $\leq k$ Durfee squares generates $w_{k}(\pi)$ unrestricted partitions of $n$. So, we have proved

Theorem 8. Let $D_{j}$ denote the set of all partitions into exactly $j$ successive Durfee squares. Then

$$
p(n)=\sum_{\substack{\sigma(\pi)=n \\ \pi \in D_{1} \cup D_{2} \cup \cdots \cup D_{k}}} w_{k}(\pi) .
$$

Since $w_{k}(\pi)=1$ for $\pi \in D_{1} \cup D_{2} \cup \cdots \cup D_{k-1}$, it follows from Theorems 7 and 8 that

$$
\sum_{\substack{\sigma(\pi)=n \\ \pi \in D_{k}}} w_{k}(\pi)=p(n)-\left|D_{1} \cup D_{2} \cup \cdots \cup D_{k-1}\right|=p(n)-\rho_{k, k}(n) .
$$

\section{Distinct Parts and Rogers-Ramanujan partitions}

In this section we obtain a weighted identity connecting $P_{T}(n)=Q(n)$ and $P_{S}(n)=\rho(n)$ (see Theorems 9 and 10 below). We first prove these identities combinatorially and then obtain their generating function form.

Consider the product

$$
\left(-a q ; q^{2}\right)_{\infty}\left(-b q^{2} ; q^{2}\right)_{\infty}
$$

as the generating function for partitions $\pi$ into distinct parts, where $\pi$ is written here as a bipartition $\left(\pi_{1}, \pi_{2}\right)$ with $\pi_{1}$ having distinct odd parts and $\pi_{2}$ having distinct even parts. The partition $\left(\pi_{1}, \pi_{2}\right)$ is counted with weight $a^{\nu\left(\pi_{1}\right)} b^{\nu\left(\pi_{2}\right)}$. In what follows several constructions will be described and illustrated with

$$
\pi_{1}: 17+15+11+7+3, \quad \pi_{2}: 20+18+14+8+6+2 .
$$

Step 1 (Decomposition). Decompose $\pi_{2}$ as $\pi_{4} \cup \pi_{5}$, where $\pi_{4}$ consists of the parts of $\pi_{2}$ which are $\leq 2 \nu\left(\pi_{1}\right)$ and $\pi_{5}$ has the remaining parts of $\pi_{2}$ (each $>2 \nu\left(\pi_{1}\right)$ ):

$$
\pi_{4}: 8+6+2, \quad \pi_{5}: 20+18+14
$$

Step 2. Construct $\pi_{4}^{*}(2)$, the 2 -fold Ferrers conjugate of $\pi_{4}$, by either representing $\pi_{4}^{*}(2)$ as columns of twos or representing $\pi_{4}^{*}(2)$ as an ordinary Ferrers graph whose first and second columns are of equal length, whose third and fourth columns are of equal length, etc. In $\pi_{4}^{*}(2)$, the number of nodes in each pair of equal columns (equivalently, the sums of the twos in each column) will be the parts of $\pi_{4}$. Circle the two at the bottom of each column of $\pi_{4}^{*}(2)$; equivalently, circle the bottom pair of nodes in each pair of equal columns of nodes of $\pi_{4}^{*}(2)$. See Figure 4.

Step 3 (Imbedding). Consider the partition $\pi_{6}=\pi_{1}+\pi_{4}^{*}(2)$ whose parts are obtained by adding the number of nodes (or weights) in the corresponding rows of $\pi_{1}$ and $\pi_{4}^{*}(2)$ :

$$
\pi_{6}: 23+19+15+9+3
$$

Notice that the weight of $\pi_{6}$ is $a^{\nu\left(\pi_{1}\right)} b^{\nu\left(\pi_{4}\right)}$. Given the partitions $\pi_{1}$ and $\pi_{4}^{*}(2)$, the partition $\pi_{6}=\pi_{1}+\pi_{4}^{*}(2)$ is uniquely defined. But given $\pi_{6}$, there are many 


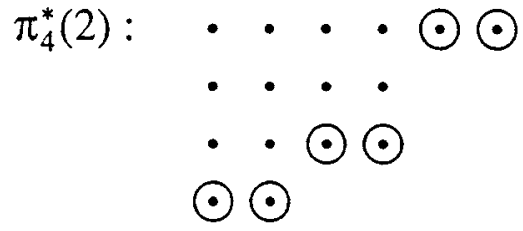

Figure 4

ways in which $\pi_{4}^{*}(2)$ can be extracted out of it. That is, there are several bipartitions $\left(\pi_{1} ; \pi_{4}^{*}(2)\right)$ which will yield the same $\pi_{6}$. We now determine all bipartitions $\left(\pi_{1} ; \pi_{4}^{*}(2)\right)$ which correspond to a specific $\pi_{6}$.

In $\pi_{6}=b_{1}+b_{2}+\cdots+b_{\nu}$ with $b_{1}>b_{2}>\cdots>b_{\nu}$, the parts $b_{l}$ which correspond to the circled nodes satisfy $b_{\ell}-b_{\ell+1} \geq 4$, where we have made the convention $b_{\nu+1}=-1$. Conversely, given a partition $\pi_{6}$ into distinct odd parts $b_{1}>b_{2}>\cdots \geq$ $b_{\nu}$, consider a part $b_{\ell}$ satisfying $b_{\ell}-b_{\ell+1} \geq 4$, with the convention $b_{\nu+1}=-1$. From every such gap $\geq 4$ we now have a choice of either extracting a pair of equal columns or not. If $b_{\ell}-b_{\ell+1}=2$, we cannot extract a pair of equal columns. Thus if $b_{\ell}-b_{\ell+1} \geq 4$, we define the weight of $b_{\ell}$ to be $a+a b=a(1+b)$. If $b_{\ell}-b_{\ell+1}=2$, we define the weight of $b_{\ell}$ to be $a$. Finally, the weight of the partition $\pi_{6}$ is the product of the weights of its parts as described above.

Step 4 . Write the parts of $\pi_{5}$ in the form of a column in descending order and below it write the parts of $\pi_{6}$ in descending order to form a column $C$. (See the top of the next page.)

Step 5. Subtract 0 from the bottom element of $C, 2$ from the next one above, 4 from the one above that, and so on, and display the new values and the subtracted values in two adjacent columns $C_{1} \mid C_{2}$.

Step 6 (Rearrangement). Rearrange the elements of $C_{1}$ in decreasing order to form a column $C_{1}^{R}$.

Step 7 . Form a partition $\pi_{3}$ by adding the corresponding elements of $C_{1}^{R}$ and $C_{2}$. Note that $\pi_{3}$ is a partition with minimal difference 2. Moreover, the number of even parts of $\pi_{3}$ equals the number of parts of $\pi_{5}$, and the number of odd parts of $\pi_{3}$ equals $\nu\left(\pi_{1}\right)=\nu\left(\pi_{6}\right)$.

\begin{tabular}{|c|}
\hline Step 4 \\
\hline$\pi_{5} / \pi_{6}$ \\
\hline 20 \\
18 \\
14 \\
\hline 23 \\
19 \\
15 \\
9 \\
3 \\
\hline
\end{tabular}

\begin{tabular}{|c|c|}
\hline \multicolumn{2}{|c|}{ Step 5} \\
\hline$C_{1}$ & $C_{2}$ \\
\hline 6 & 14 \\
6 & 12 \\
4 & 10 \\
15 & 8 \\
13 & 6 \\
11 & 4 \\
7 & 2 \\
3 & 0 \\
\hline
\end{tabular}

\begin{tabular}{|c|c|}
\hline \multicolumn{2}{|c|}{ Step 6} \\
\hline$C_{1}^{R}$ & $C_{2}$ \\
\hline 15 & 14 \\
13 & 12 \\
11 & 10 \\
7 & 8 \\
6 & 6 \\
6 & 4 \\
4 & 2 \\
3 & 0 \\
\hline
\end{tabular}

\begin{tabular}{|c|}
\hline Step 7 \\
\hline$\pi_{3}$ \\
\hline 29 \\
25 \\
21 \\
15 \\
12 \\
10 \\
6 \\
3 \\
\hline
\end{tabular}


The weight of $\pi_{3}$ is computed as follows: All even parts of $\pi_{3}$ have weight $b$. The odd parts $b_{\ell}$ of $\pi_{3}$ have weight $a$ or $a(1+b)$ depending on whether $b_{\ell}-b_{\ell+1}=2$ or $>2$. (Here again we have the convention that if $\pi_{3}=b_{1}+b_{2}+\cdots+b_{\nu}$, then $b_{\nu+1}=-1$.) The weight $w\left(\pi_{3}\right)$ of $\pi_{3}$ is the product of the weights of its parts. With this definition of weights, the combinatorial procedure described above yields the following result:

Theorem 9. Let $D$ denote the set of all partitions into distinct parts. Given $\pi \in$ $D$, let $\nu_{1}(\pi)$ denote the number of odd parts of $\pi$ and $\nu_{2}(\pi)$ the number of even parts of $\pi$. Let $R$ be the set of partitions with minimal difference 2. Then

$$
\sum_{\substack{\sigma(\pi)=n \\ \pi \in D}} a^{\nu_{1}(\pi)} b^{\nu_{2}(\pi)}=\sum_{\substack{\sigma\left(\pi_{3}\right)=n \\ \pi_{3} \in R}} w\left(\pi_{3}\right)
$$

Take $a=b=1$ in Theorem 9 to get

Theorem 10. Let $\rho_{k}(n)$ denote the number of Rogers-Ramanujan partitions of $n$ in the form $n=b_{1}+b_{2}+\cdots+b_{\nu}$ such that there are exactly $k$ odd parts $b_{\ell}$ satisfying $b_{\ell}-b_{\ell+1}>2$, with the convention $b_{\nu+1}=-1$. Then

$$
Q(n)=\sum_{k} \rho_{k}(n) 2^{k}
$$

Remark. The combinatorial method employed above in steps $1-7$ is a technique due to Bressoud but with an important variation. Bressoud [9] used the method to set up bijections between sets of partitions. For example, he used the method to give a combinatorial proof of the following result:

Göllnitz' (Little) Theorem [11]. The number of partitions of $n$ into parts $\geq 2$ with minimal difference 2 , and no consecutive odd parts, equals the number of partitions of $n$ into distinct parts $\equiv 0,2,3(\bmod 4)$.

In Bressoud's case, all the Steps 1-7 were bijections. The main variation here is that we do not require each step to be a bijection. For example, in the proof of Theorem 9 given above, Step 3, the imbedding, is not a bijection. This greater flexibility allows us to compare partition functions which are unequal, but which become equal with weights attached.

In the above proof, partitions into distinct even parts are imbedded into partitions with distinct odd parts. One could do it the other way, namely imbed distinct odd parts into distinct even parts. In this case the transition from Step 5 to Step 6 will not be a bijection, but the imbedding (Step 3) itself will be a bijection. The method of extraction of $\pi_{4}^{*}(2)$ out of $\pi_{6}$ in this case will make use of a change of parity idea as discussed in our recent paper on Capparelli's conjecture (see AlladiAndrews-Gordon [3]), where a variation in Bressoud's method is employed. In the next section another weighted identity will be discussed. There the weights arise because the transition from Step 5 to Step 6 is not a bijection.

One can obtain a generating function form of Theorem 9 by following Steps 1-7. To start with, Step 1 corresponds to the representation of the product in (5.1) in the form

$$
\left(-a q ; q^{2}\right)_{\infty}\left(-b q^{2} ; q^{2}\right)_{\infty}=\sum_{n=0}^{\infty} \frac{a^{n} q^{n^{2}}}{\left(q^{2} ; q^{2}\right)_{n}}\left(-b q^{2} ; q^{2}\right)_{n}\left(-b q^{2 n+2} ; q^{2}\right)_{\infty}
$$


At this stage it is worthwhile to introduce a third free parameter $c$ and consider the more general series

$$
\sum_{n=0}^{\infty} \frac{a^{n} q^{n^{2}}}{\left(q^{2} ; q^{2}\right)_{n}}\left(-b q^{2} ; q^{2}\right)_{n}\left(-c q^{2 n+2} ; q^{2}\right)_{\infty}
$$

which is equal to

$$
\begin{aligned}
& \sum_{n=0}^{\infty} \frac{a_{n} q^{n^{2}}}{\left(q^{2} ; q^{2}\right)_{n}}\left(-b q^{2} ; q^{2}\right)_{n} \sum_{m=0}^{\infty} \frac{c^{m} q^{2 n m} q^{m^{2}+m}}{\left(q^{2} ; q^{2}\right)_{m}} \\
= & \sum_{N=0}^{\infty} \frac{q^{N^{2}}}{\left(q^{2} ; q^{2}\right)_{N}} \sum_{m=0}^{N} c^{m} a^{N-m} q^{m}\left(-b q^{2} ; q^{2}\right)_{N-m} \frac{\left(q^{2} ; q^{2}\right)_{N}}{\left(q^{2} ; q^{2}\right)_{m}\left(q^{2} ; q^{2}\right)_{N-m}},
\end{aligned}
$$

by setting $N=m+n$. The series on the right hand side of (5.4) is the generating function for Rogers-Ramanujan partitions $\pi_{3}$ with weights more general than in Theorem 9 , as indicated in the table below.

Let $\pi_{3}: b_{1}+b_{2}+\cdots+b_{\nu}$ be a partition with minimal difference 2 and $b_{\nu+1}=-1$. Assign to each part a weight according to the following table:

$$
\begin{array}{lcl}
\underline{\text { Part }} & \text { Condition } & \text { Weight } \\
b_{l} \text { even } & - & c \\
b_{l} \text { odd } & b_{l}-b_{l+1}=2 & a \\
b_{l} \text { odd } & b_{l}-b_{l+1}>2 & a(1+b)
\end{array}
$$

The weight $w\left(\pi_{3}\right)$ of $\pi_{3}$ is the product of weights of its parts.

The series in (5.3) is the generating function for partitions $\pi$ into distinct parts counted with weights as follows. Every such partition $\pi$ is written as a vector partition $\pi=\left(\pi_{1}, \pi_{2}, \pi_{2}^{+}\right)$, where $\pi_{1}$ has distinct odd parts, $\pi_{2}$ and $\pi_{2}^{+}$have distinct even parts satisfying the conditions that $\lambda\left(\pi_{2}\right) \leq 2 \nu\left(\pi_{1}\right)$ and each part of $\pi_{2}^{+}$is > $2 \nu\left(\pi_{1}\right)$. The weight of $\pi$ is $a^{\nu\left(\pi_{1}\right)} b^{\nu\left(\pi_{2}\right)} c^{\nu\left(\pi_{2}^{+}\right)}$. This leads to a further strengthening of Theorem 9 in the form

$$
\sum_{\substack{\pi=\left(\pi_{1}, \pi_{2}, \pi_{2}^{+}\right) \\ \sigma(\pi)=n \\ \pi \in D}} a^{\nu\left(\pi_{1}\right)} b^{\nu\left(\pi_{2}\right)} c^{\nu\left(\pi_{2}^{+}\right)}=\sum_{\substack{\sigma\left(\pi_{3}\right)=n \\ \pi_{3}=R}} w\left(\pi_{3}\right)
$$

with $w\left(\pi_{3}\right)$ as described by the table above.

The weighted identities derived here have several interesting applications. The first one, although very simple, is quite elegant.

Take $a=b=c=-1$. Then the series in (5.2) and (5.3) are both equal to

$$
(q)_{\infty}=\sum_{k=-\infty}^{\infty}(-1)^{k} q^{\left(3 k^{2}-k\right) / 2}
$$

by Euler's Pentagonal Numbers Theorem. Since $a(1+b)=0$ in this case, we deduce from the weight table that the partitions we are counting are those consisting of consecutive odd parts starting from 1 , followed by even parts each greater than every odd part. This leads to 
Theorem 11. Let $P_{e}(n)$ (resp. $\left.P_{0}(n)\right)$ denote the number of partitions of $n$ into an even (resp. odd) number of distinct parts such that every even part is greater than every odd part and such that if $m$ is an odd part, then every positive odd integer $<m$ occurs as a part. Then

$$
P_{e}(n)-P_{0}(n)= \begin{cases}(-1)^{k}, & \text { if } n=\frac{3 k^{2}-k}{2}, k=0, \pm 1, \pm 2, \ldots \\ 0, & \text { otherwise. }\end{cases}
$$

The generating function form of Theorem 11 is

$$
\sum_{n=0}^{\infty}(-1)^{n} q^{n^{2}}\left(q^{2 n+2} ; q^{2}\right)_{\infty}=(q)_{\infty}=\sum_{k=-\infty}^{\infty}(-1)^{k} q^{\left(3 k^{2}-k\right) / 2} .
$$

A more interesting application is obtained by replacing $b$ by $b / a$ in the weight table so that $a(1+b)$ is replaced by $a+b$. With this change, take $a=0$. So now we are counting partitions with minimal difference 2 , each part $\geq 2$, and no consecutive odd parts, as in Göllnitz' (little) theorem. From (5.3) we see that the generating function for such partitions is equal to

$$
\begin{aligned}
& \lim _{a \rightarrow 0} \sum_{n=0}^{\infty} \frac{a^{n} q^{n^{2}}\left(-\frac{b}{a} q^{2} ; q^{2}\right)_{n}\left(-c q^{2 n+2} ; q^{2}\right)_{\infty}}{\left(q^{2} ; q^{2}\right)_{n}} \\
= & \sum_{n=0}^{\infty} \frac{b^{n} q^{2 n^{2}+n}\left(-c q^{2 n+2} ; q^{2}\right)_{\infty}}{\left(q^{2} ; q^{2}\right)_{n}} .
\end{aligned}
$$

In (5.7) take $c=1$ to get

$$
\left(-q^{2} ; q^{2}\right)_{\infty} \sum_{n=0}^{\infty} \frac{b^{n} q^{2 n^{2}+n}}{\left(q^{4} ; q^{4}\right)_{n}}=\left(-q^{2} ; q^{2}\right)_{\infty}\left(-b q^{3} ; q^{4}\right)_{\infty}
$$

leading to a refined form of Göllnitz' (little) theorem involving a free parameter $b$. This refinement is well known, but the derivation here as a limiting case $a \rightarrow 0$ is different from earlier approaches.

\section{LEBESGUE'S IDENTITY AND PARTITIONS INTO DISTINCT PARTS}

A well known identity due to Lebesgue (see Andrews [5], Ch. 2) is

$$
\sum_{n=0}^{\infty} \frac{q^{n(n+1) / 2}(-b q)_{n}}{(q)_{n}}=\left(-b q^{2} ; q^{2}\right)_{\infty}(-q)_{\infty}
$$

By Euler's identity, the product on the right hand side of (6.1) is equal to

$$
\frac{\left(-b q^{2} ; q^{2}\right)_{\infty}}{\left(q ; q^{2}\right)_{\infty}}=\sum_{n} \sum_{k} g(n, k) b^{k} q^{n}
$$

where $g(n, k)$ is the number of partitions of $n$ in which even parts do not repeat, and there are exactly $k$ even parts. If we take $b=1$ in (6.2), we get the generating function of

$$
g(n)=\sum_{k} g(n ; k)
$$

the partition function defined in $\S 1$.

Note that Göllnitz' (little) theorem is a consequence of (6.1) under

$$
q \mapsto q^{2}, b \mapsto b q^{-1}
$$


In Alladi-Gordon [4], a combinatorial proof of (6.1) was given. From the combinatorial proof the following partition theorem was deduced.

Theorem 12. Let $g(n, k)$ be as in (6.2).

Let $Q(n ; k)$ denote the number of partitions of $n$ into distinct parts $b_{1}+b_{2}+\cdots+$ $b_{\nu}$, such that there are exactly $k$ gaps $b_{i}-b_{i+1} \geq 2$, with the convention $b_{\nu+1}=0$. Then

$$
\sum_{k} g(n, k) b^{k}=\sum_{k} Q(n, k)(1+b)^{k} .
$$

In particular with $b=1$ in Theorem 12 , we obtain the weighted identity

Theorem 13. Let $w(\pi)=2^{k}$ if $\pi$ is counted by $Q(n, k)$. Then

$$
g(n)=\sum_{k} Q(n, k) 2^{k}=\sum_{\substack{\sigma(\pi)=n \\ \pi \in D}} w(\pi) .
$$

The combinatorial proof of (6.1) given in [5] considers the product in (6.1) as the generating function for bipartitions $\left(\pi_{1} ; \pi_{2}\right)$, where $\pi_{1}$ has distinct even parts and $\pi_{2}$ has distinct parts. The bipartition $\left(\pi_{1}, \pi_{2}\right)$ is counted with weight $b^{\nu\left(\pi_{1}\right)}$, explaining the left side of the identity in Theorem 12. The proof proceeds along Steps $1-7$ as indicated in $\S 5$ by imbedding the smaller parts of $\pi_{2}$ into $\pi_{1}$ after conjugation. It turns out (see [4] for details) that every step in the proof of (6.1) is a bijection except the transition from Step 5 to Step 6. The choices here give rise to the weight $(1+b)^{k}$ in Theorem 12 .

If we take $b=-1$ in Theorem 12, we get

$$
\sum_{k}(-1)^{k} g(n, k)=Q(n, 0) .
$$

Now $Q(n, 0)$ is the number of partitions of $n$ into distinct parts such that there are no gaps $\geq 2$ in the sense described above. The only partitions without such gaps are those of the form

$$
n=k+(k-1)+\cdots+1=k(k+1) / 2 .
$$

Thus we get

$$
Q(n, 0)= \begin{cases}1, & \text { if } n=\frac{k(k+1)}{2}, \\ 0, & \text { otherwise. }\end{cases}
$$

This is a combinatorial explanation of Gauss' identity

$$
\sum_{n=0}^{\infty} q^{n(n+1) / 2}=\frac{\left(q^{2} ; q^{2}\right)_{\infty}}{\left(q ; q^{2}\right)_{\infty}} .
$$

From (6.4) and Theorem 13 it follows that $g(n)$ is odd if and only if $n$ is a triangular number. Thus

$$
g(n) \equiv 0(\bmod 2)
$$

for almost all $n$.

More generally, the 2 -adic identity in Theorem 13 contains information concerning

$$
g(n) \equiv 0\left(\bmod 2^{k}\right) .
$$


It is not difficult to show using Theorem 13 that

$$
g(n) \equiv 0(\bmod 4)
$$

unless $n$ is a sum of a triangular number and a square. Thus the congruence (6.8) holds almost always.

Actually, the exceptional set where (6.8) fails is even smaller. In a forthcoming paper [2], the validity of the congruence (6.7) for almost all $n$ will be established for small values of $k$ using Theorem 13 and other identities.

\section{DISTINCT PARTS AND POWERS OF 2}

In his classic paper of 1882, Sylvester [16] proved the following identity:

$$
(-z q)_{\infty}=1+\sum_{n=1}^{\infty} \frac{z^{n} q^{\left(3 n^{2}-n\right) / 2}\left(1+z q^{2 n}\right)(-z q)_{n-1}}{(q)_{n}}
$$

Sylvester obtained (7.1) by analyzing partitions into distinct parts in terms of Durfee squares (see Andrews [7] for a proof of (7.1) and its relationship with other partition identities). This identity has several interesting consequences. For instance, by setting $z=-1$ in (7.1) we get

$$
(q)_{\infty}=1+\sum_{n=1}^{\infty}(-1)^{n} q^{\left(3 n^{2}-n\right) / 2}\left(1+q^{n}\right)=\sum_{n=-\infty}^{\infty}(-1)^{n} q^{\left(3 n^{2}-n\right) / 2},
$$

which is Euler's Pentagonal Numbers Theorem.

Our purpose here is to deduce from (7.1) a new combinatorial interpretation for partitions into distinct parts that I had observed in 1990 (see Theorems 14 and 15 below).

For this purpose consider the decomposition

$$
1+z q^{2 n}=1+z q^{n}-z q^{n}\left(1-q^{n}\right)
$$

and rewrite $(7.1)$ in the form

$$
(-z q)_{\infty}=1+\sum_{n=1}^{\infty} \frac{z^{n} q^{\left(3 n^{2}-n\right) / 2}(-z q)_{n}}{(q)_{n}}-\sum_{n=1}^{\infty} \frac{z^{n+1} q^{\left(3 n^{2}+n\right) / 2}(-z q)_{n-1}}{(q)_{n-1}}
$$

The term

$$
\frac{z^{n} q^{\left(3 n^{2}-n\right) / 2}}{(q)_{n}}
$$

is the generating function for partitions into $n$ parts with minimal difference 3 . Therefore

$$
\frac{z^{n} q^{\left(3 n^{2}-n\right) / 2}(-z q)_{n}}{(q)_{n}}
$$

is the generating function for bipartitions $\left(\pi_{1} ; \pi_{2}\right)$, where $\pi_{1}$ has exactly $n$ parts with minimal difference 3 , and $\pi_{2}$ has distinct parts $\leq n$. The bipartition $\left(\pi_{1}, \pi_{2}\right)$ is counted with weight $z^{\nu\left(\pi_{1}\right)+\nu\left(\pi_{2}\right)}=z^{n+\nu\left(\pi_{2}\right)}$.

Next we consider the imbedding of $\pi_{2}^{*}$, the Ferrers conjugate of $\pi_{2}$, into $\pi_{1}$, namely the partition $\pi_{3}=\pi_{1}+\pi_{2}^{*}$ obtained by adding the number of nodes in the corresponding rows of $\pi_{1}$ and $\pi_{2}^{*}$. As in the imbeddings considered above, we circle the bottom node of each column of $\pi_{2}^{*}$. Let $\pi_{3}=b_{1}+b_{2}+\cdots+b_{n}$. Note that if $\pi_{2}$ has $k$ parts, then $\pi_{2}^{*}$ has $k$ circled nodes. Thus $\pi_{3}$ has $k$ parts corresponding to 
these circled nodes. Every such part $b_{\ell}$ satisfies $b_{\ell}-b_{\ell+1} \geq 4$ if $\ell<n$, and $b_{\ell} \geq 2$ if $\ell=n$. Whereas every pair $\left(\pi_{1} ; \pi_{2}^{*}\right)$ produces a unique $\pi_{3}$, several bipartitions $\left(\pi_{1} ; \pi_{2}^{*}\right)$ can be extracted out of a given $\pi_{3}$. In other words, given a partition $\pi_{3}$ with exactly $k$ gaps $\geq 4$ and least part equal to 1 or with $(k-1)$ gaps $\geq 4$ and least part $\geq 2$, we have a choice of either extracting or not extracting columns of $\pi_{2}^{*}$ from positions where these gaps occur. Thus every partition with minimal difference 3 having either $k$ gaps $\geq 4$ and with 1 as the least part, or $k-1$ gaps $\geq 4$ and least part $\geq 2$, is counted with weight $z^{n}(1+z)^{k}$.

On the other hand, the term

$$
\frac{q^{\left(3 n^{2}+n\right) / 2}}{(q)_{n-1}}
$$

is the generating function for partitions $\pi_{1}$ into $n$ parts with minimal difference 3 and with smallest part 2 . Thus

$$
\frac{q^{\left(3 n^{2}+n\right) / 2}(-z q)_{n-1}}{(q)_{n-1}}
$$

is the generating function for bipartitions $\left(\pi_{1} ; \pi_{2}\right)$ with $\pi_{1}$ as described above, and $\pi_{2}$ having distinct parts $\leq n-1$. Now by considering the imbedding of $\pi_{2}^{*}$ into $\pi_{1}$ and its extraction we deduce that

$$
\frac{z^{n+1} q^{\left(3 n^{2}+n\right) / 2}(-z q)_{n-1}}{(q)_{n-1}}
$$

is the generating function for partitions $\pi_{3}=b_{1}+b_{2}+\cdots+b_{n}$ into $n$ parts with minimal difference 3 , with $b_{n}=2$, such that if $\pi_{3}$ has exactly $k-1$ gaps $b_{\ell}-b_{\ell+1} \geq 4$ for $1 \leq \ell<n$, then $\pi_{3}$ is counted with weight

$$
z^{n+1}(1+z)^{k-1} \text {. }
$$

Finally, observe that partitions $\pi_{3}$ with minimal difference 3 and with smallest part either 1 or $\geq 3$ are counted by the generating function in (7.5) and not in (7.6). The partitions with minimal difference 3 and smallest part $b_{n}=2$ are counted by both (7.5) and (7.6). So, the weight with which such partitions are counted on the right hand side of (7.4) is

$$
z^{n}(1+z)^{k}-z^{n+1}(1+z)^{k-1}=z^{n}(1+z)^{k-1} .
$$

Thus the series on the right in (7.4) is the generating function for partitions with minimal difference 3 , such that every such partition $\pi_{3}=b_{1}+b_{2}+\cdots+b_{n}$ having $k$ gaps $b_{\ell}-b_{\ell+1} \geq 4$ (with the convention $b_{n+1}=-1$ ) is counted with weight $z^{n}(1+z)^{k}$.

We can summarize the discussion above by stating the following theorem that we just proved, which is a new interpretation of Sylvester's identity (7.1).

Theorem 14. Let $Q_{k}(n)$ denote the number of partitions of $n$ into $k$ distinct parts.

Let $g_{3}(n ; \nu, k)$ denote the number of partitions of $n$ of the form $n=b_{1}+\cdots+b_{\nu}$ into $\nu$ parts with minimal difference 3 , such that there are precisely $k$ gaps $b_{\ell}-$ $b_{\ell+1} \geq 4$, with the convention $b_{\nu+1}=-1$. Then

$$
\sum_{k} z^{k} Q_{k}(n)=\sum_{k} \sum_{\nu} z^{\nu}(1+z)^{k} g_{3}(n ; \nu, k) .
$$

In Theorem 14 take $z=1$ to get 
Theorem 15. Let

$$
g_{3}(n, k)=\sum_{\nu} g_{3}(n ; \nu, k)
$$

Then

$$
Q(n)=\sum_{k} g_{3}(n ; k) 2^{k} .
$$

We believe that Theorem 15 is the key to the combinatorial understanding of the behavior of $Q(n)\left(\bmod 2^{k}\right)$, just as Theorem 13 of $\S 6$ can be used to study $g(n)\left(\bmod 2^{k}\right)$.

It is well known in the theory of modular forms (see Serre [15]) that the coefficients $c_{n}$ of certain modular forms satisfy

$$
c_{n}=0(\bmod m)
$$

for almost all $n$, for any integer $m$. Thus one way of proving that, for a given $k$,

$$
Q(n) \equiv 0\left(\bmod 2^{k}\right)
$$

for almost all $n$, is to construct such a modular form

$$
f_{k}(z)=\sum_{n=1}^{\infty} c_{n} e^{2 \pi i n \tau}
$$

with the coefficients satisfying the condition

$$
c_{n} \equiv Q(n)\left(\bmod 2^{k}\right) .
$$

The difficulty is that there is no single modular form $f(\tau)$ which would work for all $k$. In fact an infinite family of such modular forms needs to be constructed. It was only a few years ago that Gordon provided such a construction and thus proved the striking result that for each positive integer $k$, the congruence (7.7) is valid for almost all $n$. In a forthcoming paper [13], Gordon and Ono generalize this result to $p$-regular partitions as follows.

A $p$-regular partition is one whose parts repeat $<p$ times. Thus the generating function for $Q^{(p)}(n)$, the number of $p$-regular partitions of $n$, is

$$
\sum_{n=0}^{\infty} Q^{(p)}(n) q^{n}=\prod_{j=1}^{\infty}\left(1+q^{j}+q^{2 j}+\cdots+q^{(p-1) j}\right)=\frac{(q)_{\infty}}{\left(q^{p} ; q^{p}\right)_{\infty}} .
$$

Making heavy use of the theory of modular forms, Gordon and Ono [13] prove

Theorem 16. For every positive integer $k$, the congruence

$$
Q^{(p)}(n) \equiv 0\left(\bmod p^{k}\right)
$$

holds for almost all $n$.

Since $Q(n)$ is a fundamental function in the theory of partitions, it is obviously of interest to provide a combinatorial explanation for the validity of the congruence (7.7) almost always. We believe that Theorem 15 contains such combinatorial information, and in 1990 we used it study (7.7) for $k \leq 4$.

For example, to determine $Q(n)(\bmod 2)$ we need only consider the term $g_{3}(n, 0)$. Thus $Q(n)$ is odd if and only if $g_{3}(n, 0)$ is odd. Now $g_{3}(n, 0)$ is the number of partitions of $n$ in the form $b_{1}+b_{2}+\cdots+b_{\nu}$ such that the minimal difference is 3 , and that there are no gaps $b_{i}-b_{i+1} \geq 4$ with the convention $b_{\nu+1}=-1$. So this leaves us with only two choices. 
Case 1. $b_{\nu}=1, b_{i}-b_{i+1}=3,1 \leq i<\nu$.

Case 2. $b_{\nu}=2, b_{i}-b_{i+1}=3,1 \leq i<\nu$.

In Case 1 the integer $n$ being partitioned is $\left(3 \nu^{2}-\nu\right) / 2$; in Case 2 the number being partitioned is $\left(3 \nu^{2}+\nu\right) / 2$. Thus $Q(n)$ is odd if and only if $n$ is a pentagonal number.

By analyzing the 2-adic identity in Theorem 15 more closely, it can be shown (see [2] for details) that

$$
Q(n) \equiv 0(\bmod 4)
$$

unless $n=P_{k}+j^{2}$ can be written as a pentagonal number plus a perfect square. Thus the exceptional set has density zero. Actually, the exceptional set is even thinner than the one given above, as is shown in [2].

As $k$ gets larger, the determination of the exceptional sets using Theorem 15 becomes more and more complicated, and in [2] we investigate the cases $k \leq 4$. Involved in the study are the properties of various arithmetical functions like the divisor function.

Theorem 15 has many other implications. For instance, it can be refined by introducing three free parameters, as is shown in [1]. It is such a refinement that leads to a combinatorial correspondence related to a very deep theorem of Göllnitz [11]. Another consequence of this three parameter refinement is a new interpretation for Jacobi's triple product identity. In view of the importance of this refinement, a detailed discussion of this is done separately [1].

\section{ACKNOWLEDGEMENTS}

I would like to thank George Andrews for arranging my visit to The Pennsylvania State University in the Fall of 1994, where a portion of this work was done. Thanks are also due to Basil Gordon and Ken Ono for comments related to $§ 7$. Finally, I wish to acknowledge the helpful suggestions of the referee.

\section{REFERENCES}

1. K. Alladi, A combinatorial correspondence related to Göllnitz' (big) partition theorem and applications, Trans. Amer. Math. Soc. (to appear).

2. K. Alladi, On the divisibility of certain partition functions by small powers of 2 (in preparation).

3. K. Alladi, G. E. Andrews and B. Gordon, Refinements and generalizations of Capparelli's conjecture on partitions, J. Algebra 174 (1995), 636-658. MR 96b:11136

4. K. Alladi and B. Gordon, Partition identities and a continued fraction of Ramanujan, J. Comb. Th. Ser. A 63 (1993), 275-300. MR 94f: 11108

5. G. E. Andrews, The theory of partitions, Encyclopedia of Mathematics and its Applications, Vol. 2, Addison-Wesley, Reading (1976). MR 58:27738

6. G. E. Andrews, Partitions and Durfee dissection, Amer. J. Math. 10 (1979), 735-742. MR 80h: 10020

7. G. E. Andrews, Partitions: Yesterday and Today, New Zealand Math. Soc., Wellington (1979). MR 81b:01015

8. D. Bowman, Partitions with numbers in their gaps, Acta Arith. 74 (1996), 97-105. MR 96m:11090

9. D. M. Bressoud, On a partition theorem of Göllnitz, J. Reine Angew. Math. 305 (1979), 215-217. MR 80a:10027

10. F. Garvan, Generalizations of Dyson's rank and non-Rogers-Ramanujan partitions, Manus. Math 84 (1994), 343-359. MR 95e:11115 
11. H. Göllnitz, Partitionen with Differenzenbedingungen, J. Reine Angew. Math 225 (1967), 154-190. MR 35:2848

12. B. Gordon, A combinatorial generalization of the Rogers-Ramanujan identities, Amer. J. Math. 83 (1961), 393-399. MR 23:A809

13. B. Gordon and K. Ono, Divisibility of certain partition functions by powers of primes, The Ramanujan Journal 1 (1997), 25-34.

14. G. H. Hardy and E. M. Wright, An introduction to the theory of numbers, 4th ed., Clarendon Press, Oxford (1960). MR 81i:10002 (5th ed.)

15. J. P. Serre, Divisibilité des coefficients des formes modulaires de poids entiers, C. R. Acad. Sci. Paris A 279 (1974), 679-682. MR 52:3060

16. J. J. Sylvester, A constructive theory of partitions arranged in three Acts, an Interact and an Exodion, Amer. J. Math. 5 (1882), 251-330.

Department of Mathematics, University of Florida, Gainesville, Florida 32611

E-mail address: alladi@math.ufl.edu 